\title{
What Is the Effect of Body Mass Index on Subjective Outcome Following Vaginal Hysterectomy for Prolapse?
}

\author{
Thomas Gray ${ }^{1}$, John Money-Taylor ${ }^{2}$, Weiguang Li², Andrew G Farkas ${ }^{1}$, Patrick C. Campbell ${ }^{3}$, Stephen C. Radley \\ ${ }^{1}$ Department of Urogynaecology, Sheffield Teaching Hospitals, Sheffield, UK \\ ${ }^{2}$ University of Sheffield Medical School, Sheffield, UK \\ ${ }^{3}$ Belfast City Hospital, Belfast, UK
}

Purpose: Obesity is a significant risk factor for pelvic organ prolapse (POP), but the effects of obesity on outcomes of surgery for POP are poorly understood. The aim of this study was to assess the relationship between POP symptomatology, subjective outcomes of surgery and body mass index (BMI) in women undergoing vaginal hysterectomy for POP.

Methods: Pre- and postoperative data from a validated pelvic floor questionnaire (electronic Personal Assessment Questionnaire-Pelvic Floor) were collected prospectively from 60 women undergoing vaginal hysterectomy for POP. Of these, 20 were normal weight (BMI 18.5-24.9 kg/m²), 20 were overweight (BMI 25-29.9 kg/m²), and 20 were women with obesity (BMI 30$34.9 \mathrm{~kg} / \mathrm{m}^{2}$ ). The relationship between BMI and symptom scores for prolapse, impact on vaginal symptoms on quality of life (VS-QoL) and 'overall change in condition' was assessed. Pre- and postoperative symptom scores were compared using repeated mixed analysis of variance test for BMI as a categorical variable (normal, overweight, and obese). Spearman rank order correlation test was carried out to evaluate BMI as a continuous variable. All women underwent vaginal hysterectomy using a standardized technique.

Results: Overall, $93 \%$ of women reported improvement in their condition. The main finding was that 'overall change in condition' was negatively correlated with increasing BMI $\left(r_{s}=-0.324, P=0.028\right)$. Irrespective of BMI, significant improvements were observed in symptoms of prolapse and VS-QoL at 3-month postoperation.

Conclusions: With increasing BMI, women are likely to report lower levels of satisfaction following prolapse surgery, despite reporting equivalent improvements in symptoms. BMI is known to affect how individuals perceive their general health and well-being with obese individuals reporting poorer levels of subjective health status. Women with obesity may perceive change in their condition after prolapse surgery differently to women of normal weight. Reduction of weight prior to prolapse surgery could be considered in obese women to improve subjective outcomes of surgery.

Keywords: Obesity; Patient outcome assessment; Pelvic organ prolapse; Hysterectomy

- Research Ethics: This study was approved by the Institutional Review Board of the University of Sheffield and registered as a student project (registration number: 006343). All participants provided consent for the use of their data for research via ePAQ-PF.

- Conflict of Interest: Professor Stephen Radley is a director and shareholder of ePAQ systems limited, an NHS spin-out technology company (www.epaq.co.uk). The other authors declare they have no conflicts of interest. reported.

\section{INTRODUCTION}

Pelvic organ prolapse (POP) is a common condition for which the estimated lifetime risk of undergoing surgery is $12.6 \%$ [1]. Despite the global rise in the prevalence of obesity, defined as body mass index (BMI) greater than or equal to $30 \mathrm{~kg} / \mathrm{m}^{2}$, little
Corresponding author: Thomas Gray (iD https://orcid.org/0000-0001-7719-4366 Department of Urogynaecology, Sheffield Teaching Hospitals, c/o Tricia Kenyon, Level 4, Jessop Wing, Tree Root Walk, Sheffield, S10 2SF, UK

E-mail: Thomas.Gray@doctors.org.uk / Tel: +1447763686015

Submitted: January 12, 2019 / Accepted after revision: May 1, 2019
This is an Open Access article distributed under the terms of the Creative Commons Attribution Non-Commercial License (http://creativecommons.org/licenses/by-nc/4.0/) which permits unrestricted non-commercial use, distribution, and reproduction in any medium, provided the original work is properly cited. 
is known regarding the relationship between obesity and surgical outcomes in this field [2].

An association between increasing obesity and prevalence of prolapse has been reported by several authors, including data from a number of large population-based surveys [3-8]. Obesity is recognized as a risk factor for progression of POP [9] and is the strongest identified risk factor for posthysterectomy vaginal vault prolapse [10].

The impact of obesity on outcomes of prolapse surgery has been reported in relatively few studies. Anatomical and functional outcomes following abdominal sacrocolpopexy do not appear to be affected by obesity [11-13], whereas a study of women undergoing sacrospinous ligament fixation with anterior mesh repair reported less improvement in prolapse symptoms in the obese group [14].

The increasing prevalence of obesity is likely to lead to increasing prevalence of symptomatic POP. Improving our understanding of how obesity relates to perception and reporting of symptoms, impact on quality of life and outcomes of surgery will help to inform decision making and ensure that both clinicians and patients have a good understanding and realistic expectations of outcome. To our knowledge, the relationship between obesity and outcome following vaginal hysterectomy for uterine prolapse has not been reported. The aim of the present study was to compare subjective outcomes of surgery in women of normal weight, overweight and obesity, undergoing vaginal hysterectomy for POP, using prospectively collected data from a validated pelvic floor symptom questionnaire (electronic Personal Assessment Questionnaire-Pelvic Floor, ePAQ-PF) [15].

\section{MATERIALS AND METHODS}

The study included all women undergoing vaginal hysterectomy at Sheffield Teaching Hospitals NHS Foundation Trust, UK, during the 3-year study period who completed ePAQ-PF preoperatively and 3 months postoperatively, with documented $\mathrm{BMI}$ and patient consent for their data to be used for research.

Women who attend our unit complete the ePAQ-PF questionnaire as part of routine assessment. This provides detailed symptom and health-related quality of life (HRQoL) data, as well as recording BMI and patient consent for use of their data in research and service evaluation.

The ePAQ-PF is a validated Web-based interactive questionnaire which provides an in-depth evaluation of a woman's pelvic floor symptoms and their impact upon her HRQoL $[15,16]$.
The questionnaire can be completed online, or using a touchscreen computer terminal in clinic. The questionnaire presents up to 132 items and was designed to improve communication and detailed assessment by providing patients with an opportunity to report symptoms of an intimate and sensitive nature, many of which might be difficult to express face-to face $[17,18]$. The questionnaire covers dimensions of urinary, bowel, vaginal and sexual function.

For this study, the following items from ePAQ-PF questionnaire were assessed.

Comparison of pre- and postoperative vaginal prolapse domain scores and impact. Domain score is a composite of responses to the following questions on the ePAQ questionnaire;

- Q84: Do you feel that something is dropping down inside your lower abdomen or vagina?

- Q85: Do you feel that your vagina is too loose or too lax?

- Q86: Do you feel a lump or bulge coming down in your vagina?

- Q87: Do you feel a lump or bulge that comes out of your vagina altogether, so that you can feel it or see it on the outside?

Response options are 'never', 'occasionally', 'most of the time', or 'all of the time' and scored $0,1,2$, or 3 respectively. Symptom impact is graded as 'not a problem', 'a bit of a problem', 'quite a problem', or 'a serious problem' and scored $0,1,2$, or 3 respectively. Domain scores are calculated by dividing the sum of all item scores in the domain by the maximum total possible score and multiplying this by 100 , to produce a scale ranging from 0 to 100 . On this scale, a score of 0 indicates the best and 100 indicates the worst possible health status.

Analysis of pre- and postoperative responses to individual POP symptoms relating to; 'awareness of something coming down (SCD)' (Q84), 'vaginal laxity' (Q85), 'awareness of a vaginal lump or bulge' (Q86).

Comparison of pre- and postoperative vaginal symptoms quality of life (VS-QoL) scores generated from responses to 3 questions; (1) Overall, how much do vaginal problems interfere with your enjoyment of life? (2) Do you have any vaginal problems that interfere with physical activity? (3) Do you have any vaginal problems that interfere with social activity?

Assessment of 'overall change in condition' based on a validated 7-point scale; $0=$ very much worse, $1=$ much worse, $2=\mathrm{a}$ little worse, $3=$ no change, $4=$ a little better, $5=$ much better, $6=$ very much better.

Four surgeons carried out the hysterectomies included in this study, using a standardized technique. All women under- 
going vaginal hysterectomy and vaginal wall repair had a procedure using absorbable suture materials only. No mesh inlays or nonabsorbable suture materials were used as part of surgery. All women had McCall culdoplasty to unite the uterosacral ligaments in the midline and obliterate the dead space where a potential enterocoele could form. The uterosacral ligaments were also fixed to the vault and tied across the midline. Concomitant native tissue anterior vaginal wall repair was undertaken if point Aa on the POP quantification (POP-Q) grid was within $1 \mathrm{~cm}$ of the hymenal ring. Likewise, concomitant native tissue posterior wall repair was undertaken if point Ap on the POP-Q grid was within $1 \mathrm{~cm}$ of the hymenal ring. All patients who had a sacrospinous fixation had this undertaken with a Capio SLIM Suture Capturing Device (Boston Scientific, Marlborough, MA, USA) and 2.0 PDS was the suture material used.

Ethical approval was obtained from The University of Sheffield (registration number: 006343). Data were anonymized and statistical analysis was undertaken using IBM SPSS Statistics ver. 22.0 (IBM Co., Armonk, NY, USA). Pre- and postoperative symptom scores were compared using a repeated mixed analysis of variance (ANOVA) test for BMI as a categorical variable (normal: 18-24.9, overweight: 25-29.9, and obese: 30$35 \mathrm{~kg} / \mathrm{m}^{2}$ ). Spearman rank order correlation test was also carried out to evaluate BMI as a continuous variable.

\section{RESULTS}

In total 60 women were included in the study and completed ePAQ-PF both preoperatively and 3 months post operatively during the study period. Of these 60 women, 20 were of normal weight, 20 were overweight, and 20 were women with obesity. The average age for the cohort was 63 years (range, 37-88 years). The average age in the normal BMI group was 63.2, in the overweight group it was 61.6 and in the obese group it was 64.8. The average parity for the cohort was 2.4. The average parity in the normal BMI group was 2.4, in the overweight group it was 2.5 and in the obese group it was 2.35 . When assessed by Student t-test, there was no significant difference in age for women with normal BMI compared with overweight BMI $(\mathrm{P}=0.24)$, normal BMI compared to obese BMI $(\mathrm{P}=0.33)$, and overweight $\mathrm{BMI}$ compared with obese BMI $(\mathrm{P}=0.13)$. There was also no significant difference in parity when assessed by Student t-test for women with normal BMI compared with overweight $\mathrm{BMI}(\mathrm{P}=0.37)$, normal $\mathrm{BMI}$ compared to obese BMI $(\mathrm{P}=0.42)$, and overweight BMI compared with obese BMI
$(\mathrm{P}=0.30)$. All women in the study had uterine prolapse grade 2 or more using the POP-Q system. There was no statistically significant difference in age, parity or grade of prolapse between the 3 groups. Characteristics of the 3 BMI groups are reported in Table 1.

Fifty-six women underwent vaginal hysterectomy with anterior or posterior repair, 2 of whom underwent concomitant sacrospinous ligament fixation. Four women underwent vaginal hysterectomy, without vaginal wall repair. No other concomitant procedures were undertaken. All the surgical procedures undertaken are reported in Table 1.

\section{VS-QoL Scores}

The preoperative impact of prolapse on VS-QoL was significantly less in women with obesity when compared with women in the overweight group $(\mathrm{P}=0.026)$ and normal weight group $(\mathrm{P}=0.032)$ (Table 2). Significant improvements were observed in VS-QoL scores postoperatively in all BMI groups (Table 2). There was no significant difference in degree of improvement between different $\mathrm{BMI}$ groups $(\mathrm{P}=0.102)$.

\section{Overall Change in Condition}

Forty-six women (77\%) answered the global rating of outcome question, of whom 93\% (43 of 46) reported improvement in their condition. One woman felt that there was 'no change' and 2 women felt that their condition was 'a little worse'. Surgery resulted in improvement in overall condition in all groups: normal weight group (4.9 \pm 0.3$)$, overweight group (4.6 \pm 0.9 ), and obese group (4.5 \pm 0.8$)$. Analysing BMI as a continuous variable, BMI was significantly correlated with global rating of outcome; women with the higher BMI reporting poorer overall outcome (Spearman rank-order correlation $\mathrm{r}_{\mathrm{s}}=-0.324, \mathrm{P}=0.028$ ).

\section{Prolapse Symptoms Domain Scores}

Significant improvement was seen in the prolapse symptoms domain score for all BMI groups (Table 3). Data from all patients combined $(n=60)$ showed $91 \%$ improvement, from $55 \pm 18$ to $6 \pm 14(\mathrm{P}<0.0005)$. There was no significant difference in the degree of improvement of prolapse symptoms with increasing BMI (repeated mixed ANOVA test, $\mathrm{P}=0.57$, Spearman rank-order correlation test $\left(r_{s}=-0.104, P=0.44\right)$. There was no significant correlation between pre- and postoperative prolapse impact scores and BMI (repeated mixed ANOVA test, $\mathrm{P}=0.223$. 
Table 1. Characteristics of patients in normal BMI, overweight BMI, and obese BMI groups

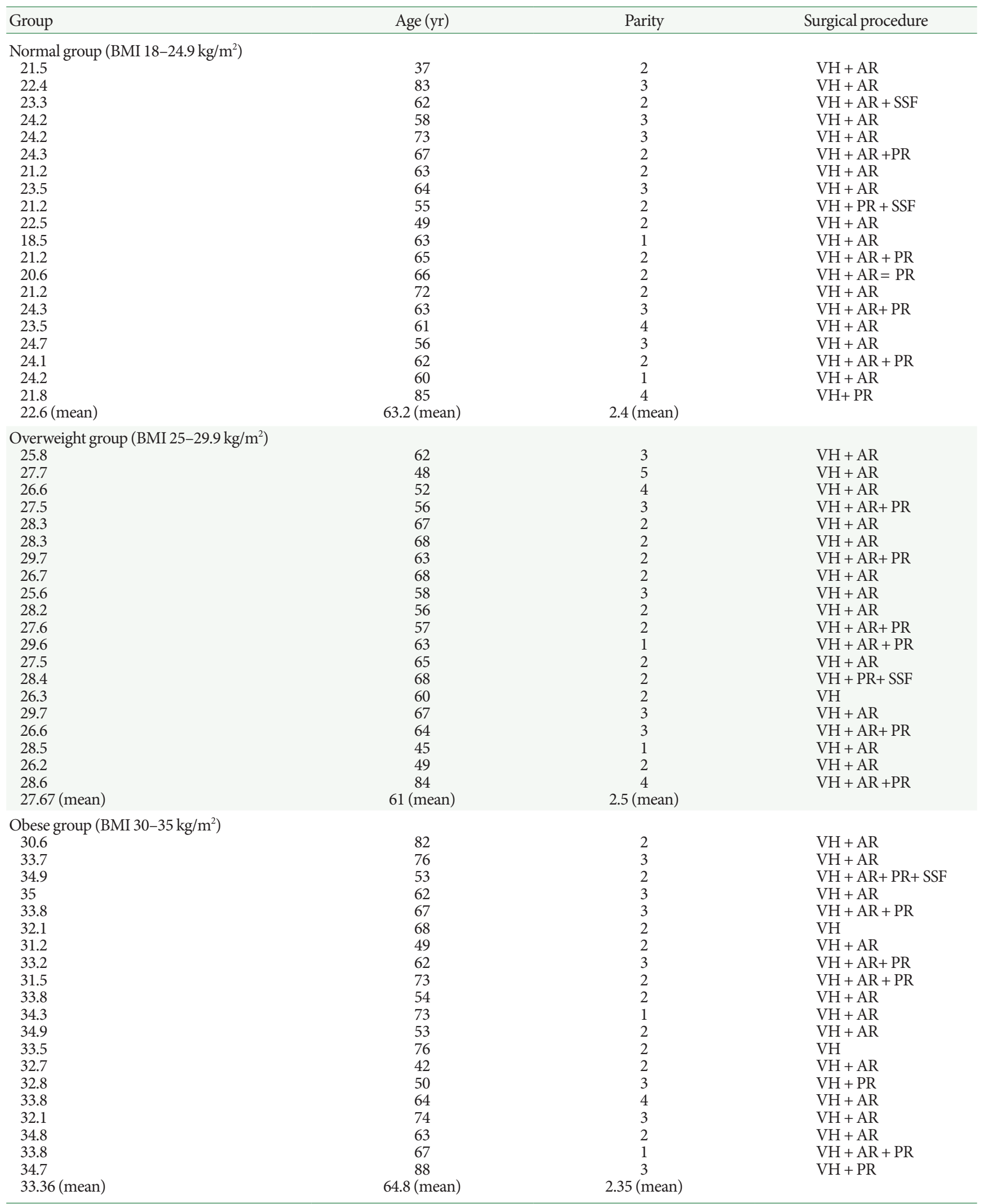

BMI, body mass index; VH, vaginal hysterectomy; AR, anterior vaginal wall repair; PR, posterior vaginal wall repair; SSF, sacrospinous fixation. 
Table 2. Pre- and postoperative vaginal symptoms quality of life scores

\begin{tabular}{lccccc}
\hline BMI group & Preoperative score & Postoperative score & Change in score & $\begin{array}{c}\text { Percentage } \\
\text { improvement }\end{array}$ & P-value \\
\hline Normal weight $(\mathrm{n}=20)$ & $57 \pm 27$ & $10 \pm 24$ & $47 \pm 39$ & 82 & $<0.0005$ \\
Overweight $(\mathrm{n}=20)$ & $57 \pm 26$ & $10 \pm 22$ & $47 \pm 34$ & 82 & $<0.0005$ \\
Obese $(\mathrm{n}=20)$ & $31 \pm 26$ & $5 \pm 15$ & $26 \pm 29$ & 84 & $<0.0005$ \\
\hline
\end{tabular}

Values are presented as mean \pm standard deviation.

BMI, body mass index.

Table 3. Pre- and postoperative prolapse symptoms domain score for each BMI group

\begin{tabular}{|c|c|c|c|c|c|}
\hline BMI group & Preoperative score & Postoperative score & Change in score & $\begin{array}{l}\text { Percentage } \\
\text { improvement }\end{array}$ & P-value \\
\hline Normal weight $(\mathrm{n}=20)$ & $60 \pm 16$ & $6 \pm 18$ & $54 \pm 25$ & $90 \%$ & $<0.0005$ \\
\hline Overweight $(\mathrm{n}=20)$ & $55 \pm 16$ & $5 \pm 13$ & $50 \pm 22$ & $91 \%$ & $<0.0005$ \\
\hline Obese $(n=20)$ & $51 \pm 21$ & $7 \pm 13$ & $45 \pm 25$ & $88 \%$ & $<0.0005$ \\
\hline
\end{tabular}

Values are presented as mean \pm standard deviation.

BMI, body mass index.

Table 4. Pre- and postoperative prolapse symptoms (all body mass index groups combined, $\mathrm{n}=60$ )

\begin{tabular}{|c|c|c|c|c|c|}
\hline Symptom & Preoperative score & Postoperative score & Change in score & $\begin{array}{l}\text { Percentage } \\
\text { improvement }\end{array}$ & P-value \\
\hline Something coming down & $2 \pm 0.7$ & $0.3 \pm 0.7$ & $1.9 \pm 0.9$ & $95 \%$ & $<0.0005$ \\
\hline Vaginal lump & $2 \pm 0.7$ & $0.3 \pm 0.8$ & $1.8 \pm 1$ & $90 \%$ & $<0.0005$ \\
\hline Vaginal laxity & $1.04 \pm 1.15$ & $0.06 \pm 0.23$ & $1 \pm 1.1$ & $96 \%$ & $<0.0005$ \\
\hline
\end{tabular}

Values are presented as mean \pm standard deviation.

Score $0=$ never 1 = occasionally; $2=$ most of the time; $3=$ all of the time.

\section{Prolapse Symptom Scores}

The 2 most commonly reported symptoms of prolapse were 'awareness of something coming down' and 'awareness of a lump', reported by $97 \%$ and $95 \%$ of women respectively. 'Vaginal laxity' was reported by $48 \%$ of women. Data from all patients combined showed significant improvement in all symptoms (Table 4). Further analysis demonstrated no significant difference in degree of improvement of individual prolapse symptoms between different BMI groups (repeated mixed ANOVA tests, $\mathrm{P}>0.05$ ).

\section{DISCUSSION}

The aim of this study was to investigate the impact of BMI on subjective outcomes of vaginal hysterectomy for POP. The main finding is that patient-reported perception of overall improvement in their condition following hysterectomy for prolapse is negatively correlated with increasing BMI.

As women with raised BMI appear to be less satisfied with subjective outcome of surgery for POP, the potential merits of delaying surgery on patients with significantly raised BMI and POP should be considered. The increased intra-abdominal pressure associated with obesity which leads to associated pressure on the pelvic floor is the theoretical mechanism implicating obesity as a risk factor for POP. Therefore, weight loss should lead to improvement in symptomatic prolapse and as this study shows, may also lead to improved subjective outcomes following surgery after weight loss.

However, the impact of weight loss on POP still remains unclear. As part of the Women's Health Initiative trial, which investigated health issues in women taking hormone replacement therapy; 16,608 women were followed up over a 5-year period to investigate the relationship between weight change and POP [9]. Although this study observed an association between 
weight gain and POP progression, weight loss was not associated with prolapse regression but in fact with borderline worsening of uterine prolapse [9]. Likewise, the impact of bariatric surgery on POP has demonstrated conflicting results, with one study reporting significant reduction in prevalence of symptoms of POP from $54 \%$ to $18 \%$ at 1-year follow-up [19], while other studies have shown no effect [20-22]. In contrast, a weight loss program for women with stress urinary incontinence reported (SUI) that women who lost $5 \%-10 \%$ of their body weight were significantly more likely to achieve substantial reduction in SUI symptoms than women in a control group [23]. There is also strong evidence that bariatric surgery has significantly beneficial effects on SUI [24].

A common rationale for delaying surgery in morbidly obese patients with POP whilst they lose weight is to reduce the risk of intraoperative complications. However, only one study has reported complications of prolapse surgery specifically related to BMI, finding that obesity was protective against blood loss, transfusion and long-term urinary retention [25]. The retrospective design of this study may have introduced selection bias as it possible that women with obesity may have been managed differently. Evidence of a significant increase in risks of immediate complications during prolapse surgery in obese patients is lacking.

A further potential rationale for recommending weight loss to patients with POP prior to surgery, is to reduce the risks of recurrence. A small retrospective study of 69 women found that BMI was a significant risk factor for surgical failure at 1 year [26] and obesity has been associated with significant risk of recurrent anterior vaginal wall prolapse following anterior colporrhaphy [27]. Conversely, a 5-year prospective study of 376 women undergoing prolapse and incontinence surgery found no association between obesity and recurrence [28]. Moreover, a systematic review from 2015 found that though higher BMI was a significant risk factor for primary POP, it was not a significant risk factor for recurrent prolapse [29]. Therefore, delaying surgery for POP whilst patients lose weight may not be beneficial for reducing complications or recurrence of the prolapse.

Comparison of our findings with other studies is limited, as only a few papers have reported the impact of obesity on outcomes of prolapse surgery. Obesity does not appear to affect anatomical or functional outcomes in women undergoing abdominal sacrocolpopexy [11-13]. A study involving women undergoing sacrospinous ligament fixation with anterior mesh, employing Asian BMI categories (where obesity is classed as
BMI $>27.5 \mathrm{~kg} / \mathrm{m}^{2}$ ), reported less symptomatic improvement in women with obesity [14]. Whereas these studies involved the use of synthetic mesh to treat vault prolapse, the present study related specifically to women undergoing vaginal hysterectomy for prolapse, so results are not directly comparable.

Since increasing BMI was associated with a lesser degree of overall improvement in condition and improvement in POP symptoms was not related to BMI, perception of outcome may be related to factors other than impact of prolapse surgery on prolapse symptoms. BMI affects how individuals perceive their general health and well-being [30] and obesity is associated with impaired health related quality of life and poor levels of subjective health status [31]. A population survey of 2,000 people in America reported decreased HRQoL with increasing obesity, even without the presence of chronic disease [31], and in a population survey of almost 10,000 people in Australia who completed the Short Form Health Survey, high BMI was associated with reduced levels of physical and mental well-being [32]. The assessment of 'overall change in condition' in the present study is a subjective measure of treatment outcome. Women with obesity may perceive change in their condition differently to women of normal weight.

The ePAQ VS-QoL score is generated from responses to questions relating to the impact of vaginal symptoms on physical and social activity and enjoyment of life. Women with obesity and POP reported significantly less impact of prolapse on their VS-QoL preoperatively than normal weight or overweight patients, a possible explanation for this being a relationship between physical activity and BMI. Women with obesity may be less likely to engage in physical activity than women of normal weight and therefore report less impact of prolapse on their quality of life. This finding may relate specifically to prolapse as it contrasts with published reports of impaired health related quality of life with increasing BMI.

The main limitations of our study are the short follow-up period (3 months) and lack of pre- and postoperative objective data, including POP-Q scores. It is possible that patients who were unsatisfied with their surgery may have disengaged from the follow-up process and not completed the postoperative questionnaire that was requested, thereby introducing reporting bias. However, our comprehensive pre- and postoperative questionnaire data from 60 patients undergoing vaginal hysterectomy for prolapse has permitted a detailed comparison of subjective outcomes between different BMI groups in this small study. 
In conclusion, we suggest that with increasing BMI, women are likely to be satisfied to a lesser degree with the outcome of prolapse surgery. This may have important implications in preoperative counselling and management of patients. Future studies should investigate the impact of obesity on outcomes of surgery for anterior and posterior compartment prolapse, assessing both subjective and objective outcomes, with long-term follow-up to assess the impact of BMI on recurrence.

\section{AUTHOR CONTRIBUTION STATEMENT}

- Full access to all the data in the study and takes responsibility for the integrity of the data and the accuracy of the data analysis: $T G G$

- Study concept and design: PCC, SCR, TGG

- Acquisition of data: PCC, JMT, TGG

- Analysis and interpretation of data: PCC, TGG, JMT, W Li

- Drafting of the manuscript: $P C C, T G$

- Critical revision of the manuscript for important intellectual content: AGF, SCR

- Statistical analysis: $W \mathrm{Li}$

- Obtained funding: $P C C, T G G$

- Administrative, technical, or material support: JMT

- Study supervision: SCR, AGF

\section{REFERENCES}

1. Wu JM, Matthews CA, Conover MM, Pate V, Jonsson Funk M. Lifetime risk of stress urinary incontinence or pelvic organ prolapse surgery. Obstet Gynecol 2014;123:1201-6.

2. World Health Organization. Global database on body mass index [Internet]. Geneva (Switzerland): World Health Organization; c2019 [cited 2019 Jun 13]. Available from: http://apps.who.int/bmi/ index.jsp.

3. Uustal Fornell E, Wingren G, Kjølhede P. Factors associated with pelvic floor dysfunction with emphasis on urinary and fecal incontinence and genital prolapse: an epidemiological study. Acta Obstet Gynecol Scand 2004;83:383-9.

4. Jelovsek JE, Maher C, Barber MD. Pelvic organ prolapse. Lancet 2007;369:1027-38.

5. Hendrix SL, Clark A, Nygaard I, Aragaki A, Barnabei V, McTiernan A. Pelvic organ prolapse in the Women's Health Initiative: gravity and gravidity. Am J Obstet Gynecol 2002;186:1160-6.

6. Mant J, Painter R, Vessey M. Epidemiology of genital prolapse: observations from the Oxford Family Planning Association Study. Br
J Obstet Gynaecol 1997;104:579-85.

7. Risk factors for genital prolapse in non-hysterectomized women around menopause. Results from a large cross-sectional study in menopausal clinics in Italy. Progetto Menopausa Italia Study Group. Eur J Obstet Gynecol Reprod Biol 2000;93:135-40.

8. Miedel A, Tegerstedt G, Maehle-Schmidt M, Nyrén O, Hammarström M. Nonobstetric risk factors for symptomatic pelvic organ prolapse. Obstet Gynecol 2009;113:1089-97.

9. Kudish BI, Iglesia CB, Sokol RJ, Cochrane B, Richter HE, Larson J, et al. Effect of weight change on natural history of pelvic organ prolapse. Obstet Gynecol 2009;113:81-8.

10. Marchionni M, Bracco GL, Checcucci V, Carabaneanu A, Coccia EM, Mecacci F, et al. True incidence of vaginal vault prolapse. Thirteen years of experience. J Reprod Med 1999;44:679-84.

11. Bradley CS, Kenton KS, Richter HE, Gao X, Zyczynski HM, Weber $\mathrm{AM}$, et al. Obesity and outcomes after sacrocolpopexy. Am J Obstet Gynecol 2008;199:690.e1-8.

12. McDermott CD, Park J, Terry CL, Woodman PJ, Hale DS. Surgical outcomes of abdominal versus laparoscopic sacral colpopexy related to body mass index. J Obstet Gynaecol Can 2012;34:47-56.

13. Menzella D, Thubert T, Joubert M, Lauratet B, Kouchner P, Lefranc JP. Influence of body mass index on the outcomes of robotic-assisted laparoscopic sacrocolpopexy: a comparative retrospective study. Prog Urol 2013;23:1482-8.

14. Lo TS, Tan YL, Khanuengkitkong S, Dass AK. Surgical outcomes of anterior trans-obturator mesh and vaginal sacrospinous ligament fixation for severe pelvic organ prolapse in overweight and obese Asian women. Int Urogynecol J 2013;24:809-16.

15. Radley SC, Jones GL, Tanguy EA, Stevens VG, Nelson C, Mathers NJ. Computer interviewing in urogynaecology: concept, development and psychometric testing of an electronic pelvic floor assessment questionnaire in primary and secondary care. BJOG 2006; 113:231-8.

16. Radley SC, Jones GL. Measuring quality of life in urogynaecology. BJOG 2004;111 Suppl 1:33-6.

17. Schüssler-Fiorenza Rose SM, Gangnon RE, Chewning B, Wald A. Increasing discussion rates of incontinence in primary care: a randomized controlled trial. J Womens Health (Larchmt) 2015; 24:940-9.

18. Dua A, Jones G, Wood H, Sidhu H. Understanding women's experiences of electronic interviewing during the clinical episode in urogynaecology: a qualitative study. Int Urogynecol J 2013;24: 1969-75.

19. Cuicchi D, Lombardi R, Cariani S, Leuratti L, Lecce F, Cola B. Clinical and instrumental evaluation of pelvic floor disorders be- 
fore and after bariatric surgery in obese women. Surg Obes Relat Dis 2013;9:69-75.

20. Whitcomb EL, Horgan S, Donohue MC, Lukacz ES. Impact of surgically induced weight loss on pelvic floor disorders. Int Urogynecol J 2012;23:1111-6.

21. McDermott CD, Terry CL, Mattar SG, Hale DS. Female pelvic floor symptoms before and after bariatric surgery. Obes Surg 2012;22:1244-50.

22. Olivera CK, Herron DM, Kini SU, Vardy MD, Ascher-Walsh CJ, Garely AD, et al. Long-term quality of life and pelvic floor dysfunction after bariatric surgery. Am J Obstet Gynecol 2012;207:431.e14.

23. Wing RR, Creasman JM, West DS, Richter HE, Myers D, Burgio $\mathrm{KL}$, et al. Improving urinary incontinence in overweight and obese women through modest weight loss. Obstet Gynecol 2010;116(2 Pt 1):284-92.

24. Anglim B, O'Boyle CJ, O'Sullivan OE, O'Reilly BA. The long-term effects of bariatric surgery on female urinary incontinence. Eur J Obstet Gynecol Reprod Biol 2018;231:15-8.

25. Nam KH, Jeon MJ, Hur HW, Kim SK, Bai SW. Perioperative and long-term complications among obese women undergoing vaginal surgery. Int J Gynaecol Obstet 2010;108:244-6.
26. Díez-Calzadilla NA, March-Villalba JA, Ferrandis C, Hernández JA, Martínez-Jabaloyas JM, Chuan P, et al. Risk factors in the failure of surgical repair of pelvic organ prolapse. Actas Urol Esp 2011;35: 448-53.

27. Kawasaki A, Corey EG, Laskey RA, Weidner AC, Siddiqui NY, Wu JM. Obesity as a risk for the recurrence of anterior vaginal wall prolapse after anterior colporrhaphy. J Reprod Med 2013;58:195-9.

28. Clark AL, Gregory T, Smith VJ, Edwards R. Epidemiologic evaluation of reoperation for surgically treated pelvic organ prolapse and urinary incontinence. Am J Obstet Gynecol 2003;189:1261-7.

29. Vergeldt TF, Weemhoff M, IntHout J, Kluivers KB. Risk factors for pelvic organ prolapse and its recurrence: a systematic review. Int Urogynecol J 2015;26:1559-73.

30. Fontaine KR, Barofsky I. Obesity and health-related quality of life. Obes Rev 2001;2:173-82.

31. Jia H, Lubetkin EI. The impact of obesity on health-related qualityof-life in the general adult US population. J Public Health (Oxf) 2005;27:156-64.

32. Renzaho A, Wooden M, Houng B. Associations between body mass index and health-related quality of life among Australian adults. Qual Life Res 2010;19:515-20. 